\title{
Strain-gradient effect on the crack tip dislocations density
}

\author{
Valery Shlyannikov, Andrey Tumanov, Ruslan Khamidullin \\ FRC Kazan Scientific Center of Russian Academy of Sciences, Russia \\ shlyannikov@mail.ru,bttp:/ / orcid.org/0000-0003-2468-9300 \\ tymanoff@rambler.ru, ruslankhamidullin94@mail.cru
}

\begin{abstract}
In this study, the influence of a material's plastic properties on the crack tip fields and dislocation density behavior is analytically and numerically analyzed using the conventional mechanism-based strain-gradient plasticity (CMSGP) theory established using the Taylor model. The material constitutive equation is implemented in a commercial finite element code by a user subroutine, and the crack tip fields are evaluated with novel parameters in the form of the intrinsic material length, characterizing the scale over which gradient effects become significant. As a consequence of the strain-gradient contribution, FE results show a significant increase in the magnitude of the stress fields of CMSGP when the material length parameter is considered. It is found that the density of geometrically necessary dislocations (GND) is large around the crack tip, but it rapidly decreases away from the crack tip. On the contrary, the density of statistically stored dislocations (SSD) is not as large as geometrically necessary dislocations around the crack tip, but it decreases much slower than GND away from the crack tip. A couple effect of material work hardening and the crack tip distance is identified.
\end{abstract}

KEYWORDS. Strain gradient; Crack tip; Dislocations density.

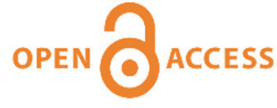

Citation: Shlyannikov, V., Tumanov, A., Khamidullin, R., Strain-gradient effect on the crack tip dislocations density, Frattura ed Integrità Strutturale, 54 (2020) 192-201.

Received: 04.08 .2020

Accepted: 27.08 .2020

Published: 01.10.2020

Copyright: (C) 2020 This is an open access article under the terms of the CC-BY 4.0 which permits unrestricted use, distribution, and reproduction in any medium, provided the original author and source are credited.

\section{INTRODUCTION}

$\mathrm{T}$ he last quarter of a century witnessed increasing attention being drawn to problems of gradient plasticity. This is attributed to the established effects of measuring characteristics on small scales with respect to the structure of the material, leading to a sufficient increase in true local stresses. Several experiments, including micro-indentation hardness and micro-torsion tests, have shown that metallic materials demonstrate a strong size effect at the micron and sub-micron scales. These size effects are attributed to geometrical dislocations induced by non uniform plastic deformation and strain gradients. Constitutive models of classical plasticity theories do not take into account the intrinsic material lengths, and thus cannot describe size-dependent material behavior at the micron scale. Fleck and Hutchinson [1,2] and Fleck et al. [3] developed a phenomenological gradient theory of the plasticity of materials and structures whose dimensions control plastic deformation, in the range of approximately a tenth of a micron to tens of microns. They have been applied to numerous problems where strain gradient effects are expected to play significant roles in the behavior of the material, including in the analysis of stress fields at the crack tip (Huang et al.[4,5], Xia and Hutchinson [6]). To enable 
dimensional matching, characteristic length $/$ was introduced to scale the components of the rotational gradient of coupled stresses (Fleck and Hutchinson,[1], Fleck et al., [3]). This length scale was considered as an internal parameter of the material structure associated with the dislocation density.

An analysis by Nix and Gao [7] partially elucidated the property embodied by the characteristic length $l$ of the material, introduced by Fleck and Hutchinson [1] and indicated the need to further refine the gradient theory of plasticity by an experimental law based on an analysis of the dominant deformation mechanisms. Nix and Gao [7] used the Taylor model to clarify the relationship between the shear strength and dislocation density of a material and identified a characteristic parameter of the material structure, which was introduced in the original formulation of the theory of gradient plasticity by Fleck and Hutchinson [1,2]. Subsequently, Gao et al. [8] supplemented the formulation which is referred to as the mechanism-based theory of strain gradient plasticity (MSG), where the characteristic length of the material structure corresponds to the scale at which the effects of the gradients are comparable with the strain values. In the presence of a large strain gradient, the total dislocation density is considered as the sum of the statistical and geometric components. The MSG theory is an attempt to establish the relationship between continuum mechanics and material science. This relationship is realized through fundamental length scales, which are combinations of elasticity and plasticity constants in combination with the Burgers vector.

Recently, Huang et al. [9] presented a simplified formulation of the gradient theory of plasticity by eliminating high-order terms associated with rotational components, naming the formulation the conventional mechanism-based strain gradient (CMSG) plasticity theory. It is likewise based on the Taylor dislocation model and preserves the structure of the classical J2 theory of plasticity. In the past two decades, applications of the CMSG gradient plasticity theory were subject to intense development to solve problems of fracture mechanics. The finite element analysis showed that the stress level in the dominant gradient plasticity zone is two to three times higher than in the classical HRR field, and the stress singularity is higher than 1/2, indicating that stresses are more singular than not in the HRR solution as well as in the classical solution with elastic stress intensity factors [10-12]. Martínez-Pañeda et al. [12-16] quantitatively determined the ratio between the parameters of the material and the physical length at which gradient effects significantly increase stresses at the crack tip. The plasticity at the crack tip is found to be suppressed when the characteristic Taylor parameter of the material structure is of the order of the size of the plastic zone, which is determined by the elastic stress intensity factor.

Gao and Huang [17] paid attention on the role of geometrically necessary dislocations in the development of continuum plasticity theories with an intrinsic material length scale. Following to this work, the purpose of our study is to investigate the crack tip dislocation behavior in CMSG plasticity.

\section{CONSTITUTIVE EQUATIONS OF CONVENTIONAL MECHANISM-BASED STRAIN GRADIENT PLASTICITY}

$\mathrm{T}$

he conventional theory of mechanism-based strain gradient plasticity (CMSG) developed by Huang et al. [9] is employed in the present study owing to the following reasons. Several authors have asserted that strain gradient plasticity theories can be classified into higher-order and lower-order theories. The first framework involves higher-order stress and therefore requires more boundary conditions; it includes the theory of mechanism-based strain gradient (MSG) plasticity established using the Taylor dislocation model. The second framework involves lower-order theories, such as the conventional theory of mechanism-based strain gradient plasticity (CMSG), which does not include a higher-order stress, where the strain gradient effect comes into play via the incremental plastic module. This is also based on the Taylor dislocation model, where the plastic strain gradient appears only in the constitutive model, and the equilibrium equations and boundary conditions are the same as those in conventional continuum theories.

According to the CMSG, the stress-strain relation in uniaxial tension is given by

$$
\sigma=\sigma_{r f} f\left(\varepsilon^{p}\right)=\sigma_{y}\left(\frac{E}{\sigma_{y}}\right)^{N}\left(\varepsilon^{p}+\frac{\sigma_{y}}{E}\right)^{N},
$$

where $\sigma_{r f}$ is a reference stress in uniaxial tension

$$
\sigma_{r e f}=\sigma_{y}\left(E / \sigma_{y}\right)^{N},
$$


and $f$ is a nondimensional function of plastic strain determined from the uniaxial stress-strain curve, which for most ductile materials can be written as a power law relation

$$
f\left(\varepsilon^{p}\right)=\left(\varepsilon^{p}+\left(\sigma_{y} / E\right)\right)^{N} .
$$

In Eqns. (1-3), $\sigma_{y}$ denotes the initial yield stress, and $N$ is the plastic work hardening exponent $(0 \leq N<1)$.

The CMSG plasticity is based on the Taylor (1938) dislocation model

$$
\tau=\alpha \mu b \sqrt{\rho}
$$

where $\tau$ is the shear flow stress, $\mu$ is the shear modulus, $b$ the Burgers vector, $\alpha$ is an empirical coefficient ranging from 0.3 to 0.5 , and $\rho$ is the dislocation density. The dislocation density $\rho$ is composed of the density $\rho_{S}$ for statistically stored dislocations (SSDs), which accumulate by trapping each other in a random manner, and density $\rho_{G}$ for geometrically necessary dislocations (GNDs), which are required for compatible deformation of various parts of the material, i.e.,

$$
\rho=\rho_{S}+\rho_{G} .
$$

The SSD density is related to the flow stress and the material stress-strain curve in uniaxial tension

$$
\rho_{S}=\left[\sigma_{r f} f\left(\varepsilon^{P}\right) / M \alpha \mu b\right]^{2}
$$

The GND density is related to the curvature of plastic deformation, or the effective plastic strain gradient $\eta^{\mathrm{P}}$, by

$$
\rho_{G}=\bar{r} \frac{\eta^{P}}{b}
$$

where $\bar{r}$ is the Nye factor, which is around 1.90 for face-centered-cubic polycrystals. The measure of the effective plastic strain gradient $\eta^{\mathrm{P}}$ was reported by Gao et al. [8] in the form of three quadratic invariants of the plastic strain gradient tensor to represent $\eta^{\mathrm{p}}$, and the coefficients were determined by three models of GNDs, i.e.,

$$
\eta^{P}=\int \dot{\eta} d t ; \dot{\eta}^{P}=\sqrt{\frac{1}{4} \dot{\eta}_{i j k}^{P} \dot{\eta}_{i j k}^{P}} ; \dot{\eta}_{i j k}^{P}=\dot{\varepsilon}_{i k, j}^{P}+\dot{\varepsilon}_{j k, i}^{P}-\dot{\varepsilon}_{i j, k}^{P}
$$

where $\dot{\varepsilon}_{i j}^{P}$ is the tensor of the plastic strain rate.

The tensile flow stress is related to the shear stress by

$$
\sigma_{\text {flow }}=M \tau=M \alpha \mu b \sqrt{\rho_{S}+\bar{r} \frac{\eta^{P}}{b}}
$$

Because the plastic strain gradient $\eta^{\mathrm{P}}$ vanishes in uniaxial tension, the density $\rho_{S}$ for SSDs is described by Eqn. (6), and the flow stress becomes

$$
\sigma_{f l o w}=\sigma_{r e f} \sqrt{f^{2}\left(\varepsilon^{P}\right)+l \eta^{P}}
$$

where 


$$
l=18 \alpha^{2}\left(\mu / \sigma_{y}\right)^{2} b
$$

is the intrinsic material length in the strain gradient plasticity based on parameters of elasticity (shear modulus $\mu$ ), plasticity (reference stress $\sigma_{r e f}$ ), and atomic spacing (Burgers vector $b$ ). For metallic materials, the internal material length is indeed on the order of microns, consistent with the estimate by Fleck and Hutchinson [2].

To avoid the uses of higher-order stresses, Huang et al. [9] proposed a viscoplastic formulation of the CMSG plasticity in the form of the following constitutive equations

$$
\begin{gathered}
\dot{\varepsilon}^{p}=\dot{\varepsilon}\left(\frac{\sigma_{e}}{\sigma_{\text {flow }}}\right)^{m}=\dot{\varepsilon}\left[\frac{\sigma_{e}}{\sigma_{r f} \sqrt{f^{2}\left(\varepsilon^{p}\right)+l \eta^{p}}}\right]^{m} \\
\dot{\sigma}_{i j}=K \dot{\varepsilon}_{k k} \delta_{i j}+2 \mu\left[\dot{\varepsilon}_{i j}^{\prime}-\frac{3 \dot{\varepsilon}}{2 \sigma_{e}}\left(\frac{\sigma_{e}}{\sigma_{\text {flow }}}\right)^{m} \sigma_{i j}^{\prime}\right],
\end{gathered}
$$

where $\sigma_{e}$ is the effective stress, $\dot{\varepsilon}_{i j}^{\prime}$ is the deviatoric strain rate, and $m$ is the rate-sensitivity exponent. Notably, Huang et al. [9] compared CMSG with the higher-order theory of mechanism-based strain gradient plasticity (Gao et al.,[8]) established from the same Taylor dislocation model. The stress distributions predicted by the lower and higher-order theories are only different within a thin boundary layer, whose thickness is approximately $10 \mathrm{~nm}$. CMSG, along with other continuum plasticity theories must have lower limits and cannot be applied down to the nanometer scale. This is because the continuum plasticity theories represent the collective behavior of discrete dislocations, and therefore the strain gradient effects are significant at a scale larger than the average dislocation spacing, such that continuum plasticity is still applicable. This lower limit, however, is not a fixed constant, and it may vary for different materials. However, such a lower limit exists, below which CMSG and other continuum plasticity theories are not applicable. There is no upper limit of CMSG, as the strain gradient term $l \eta^{P}$ becomes negligible at the large scale. CMSG then naturally degenerates to classical plasticity.

\section{SUBJECT FOR CONSIDERATION AND LOADING CONDITIONS FOR NUMERICAL FEM ANALYSES}

T he geometry considered in this study is the compact tension (CT) specimen. The CT configuration is applied conventionally for the numerical and experimental studies in fracture mechanics. The load is prescribed by imposing a displacement on the pins. We model the contact between the pins and the specimen by using a surface to surface contact algorithm with finite sliding (Fig.1).

The principal feature of our study is the evaluation of coupling material properties and strain gradient plasticity effects. To this end, the wide range of plastic work hardening exponent $N$ for the elastic-plastic solids at a specified value of the intrinsic material length parameter $l$, have been used in our calculations. In the numerical results to be considered, the comparative analysis is based on assuming different values of the normalized, remotely applied elastic stress intensity factor (SIF) $K_{1} / \sigma_{Y} \sqrt{l}$. Different pure Mode I loading conditions are obtained for considered configuration by combinations of the nominal stress level and the initial crack length. In the following, stresses $\sigma_{i j}$ are normalized by the yield stress $\sigma_{Y}$ in uniaxial tension, while the distance $r$ to the crack tip is normalized by the internal material length $l$ in CMSG plasticity. It must be pointed out that the internal material length $l$ has been used to normalize $r$ and $K_{l}$, and this intrinsic material length parameter enters the constitutive equation for dimensional consistency. The value of $l$ can be obtained by fitting micro-scale experiments and typically ranges between $1 \mu \mathrm{m}$ and $10 \mu \mathrm{m}$. The CMSGP model recovers the conventional plasticity solution when $l=0$. The crack faces for considered subject remain traction-free. The elastic stress intensity factor, $K_{1}$, of the remotely applied field increases monotonically, such that there is no unloading. 


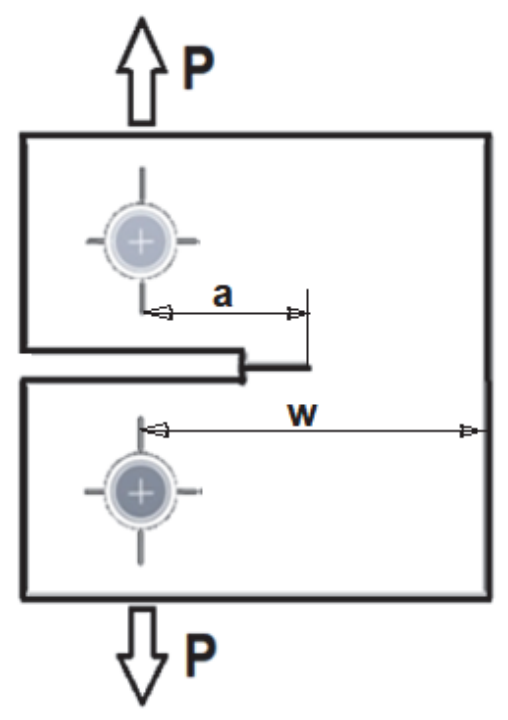

Figure 1: Compact tension specimen.

The numerical results for the CT specimen presented in this study concern the following sets of loading conditions and material properties: $\sigma_{Y} / E=0.002$, plastic work hardening exponents are $N=0.1,0.2$; the intrinsic material lengths is $l=$ $5 \mu \mathrm{m}$, external applied loads are $\bar{K}_{1}=K_{1} / \sigma_{Y} \sqrt{l}=10.87,21.73$ and the Poissons' ratio is $v=0.3$.

Unlike the higher-order theory of mechanism-based strain gradient plasticity, CMSG is a lower-order theory, which does not involve a higher-order stress, such that its governing equations are essentially the same as those in classical plasticity theories. Existing finite element programs can be easily modified to incorporate the plastic strain gradient effect. The framework presented in Section 2 is numerically implemented by using the ANSYS program [18]. Specifically, we implemented the constitutive relations (Eqns.1,12,13) of CMSG in the finite element program ANSYS via its USERMATERIAL subroutine UMAT. It only differs from classical plasticity in that the plastic strain gradient must be evaluated in UMAT. Fortran modules are used to store the plastic strain components across the Gaussian integration points, and the plastic strain gradient is computed by numerical differentiation within the element. This is accomplished by interpolating the plastic strain increment $\varepsilon_{p}$ within each element via the values at Gaussian integration points in isoparametric space, and subsequently determining the gradient of the plastic strain increment via differentiation of the shape function.

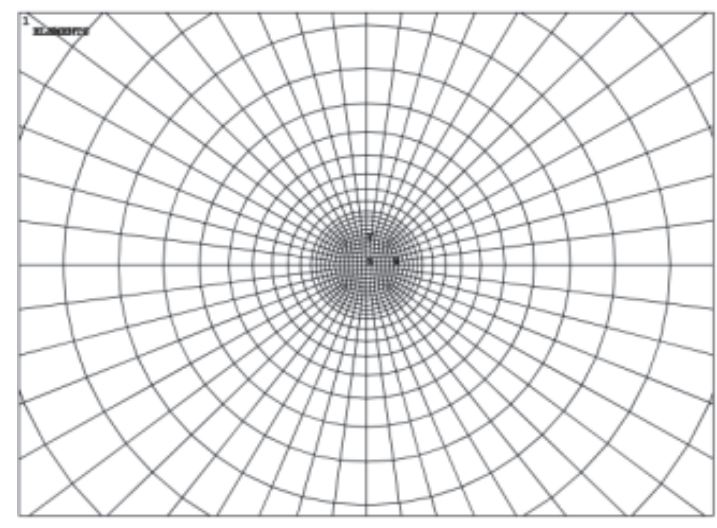

Figure 2: Crack tip finite element mesh.

We model an CT specimen (Fig.1) of width $\mathrm{W}=50 \mathrm{~mm}$ and initial crack length $a_{0}=23.65 \mathrm{~mm}$. In the finite element models, an initial crack tip is defined as a notch with finite curvature radius $\rho=0.06 \mu \mathrm{m}$. With the aim of accurately characterizing the influence of the strain gradient, a highly refined mesh is used near the crack tip. After a mesh sensitivity analysis, the size of the elements is on the order $5 \mathrm{~nm}$; the typical number of quadrilateral quadratic plane strain elements 
is approximately 550 000. As shown in Fig. 2, a very refined mesh is used near the crack tip to accurately capture the influence of plastic strain gradients. Efforts are made to ensure that the elements have an aspect ratio close to one. Mesh refinement and a comparison of different elements can ensure that the numerical results are accurate.

\section{NUMERICAL RESULTS AND DISCUSSION}

The compact tension specimen is analyzed by the two-dimensional plane strain finite element method. Fig. 3 shows the effective stress $\sigma_{e} / \sigma_{Y}$, normalized by the uniaxial yield stress $\sigma_{Y}$, versus the nondimensional distance to the crack tip, $r / l$, ahead of the crack tip predicted by CMSG theory, where $l$ is the internal material length in the strain gradient plasticity. The remotely applied stress intensity factors depict the CT specimen $\bar{K}_{1}=10.87$ and $\bar{K}_{1}=21.73$, while the plastic work hardening exponents are $N=0.2$ and 0.4 . The corresponding stress distribution in the classical HRR plasticity (without strain gradient effects) is also shown in Fig. 3. and the horizontal line of $\sigma_{\text {eqv }} / \sigma_{Y}=1$ represents plastic yielding. For the specified value of $l=5 \mu \mathrm{m}$, the above result indicates that the strain gradient effects are significant within a zone of approximately $0.3 \mathrm{r} / \mathrm{l}$. This is in agreement with the Xia and Hutchinson's [6] estimate of the size of dominance zone for the asymptotic and Jiang et al. [10] numerical crack tip fields in strain gradient plasticity. Once the distance to the crack tip is less than $0.3 \mathrm{r} / \mathrm{l}$, the effective stress predicted by CMSG plasticity increases considerably more rapidly than its counterpart in conventional HRR plasticity, which is dependent on the applied stress intensity factor level $\bar{K}_{1}$ and the plastic work hardening exponent $N$ value. At a relatively small remote stress intensity factor $\bar{K}_{1}=10.87$, the equivalent stress accounting for the strain gradient effect is approximately five times higher and more than that in classical plasticity.

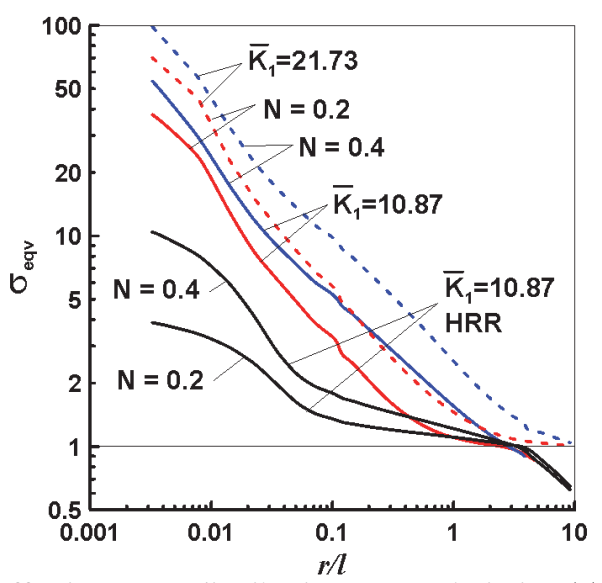

Figure 3: Effective stress distributions at crack tip in CT specimen.
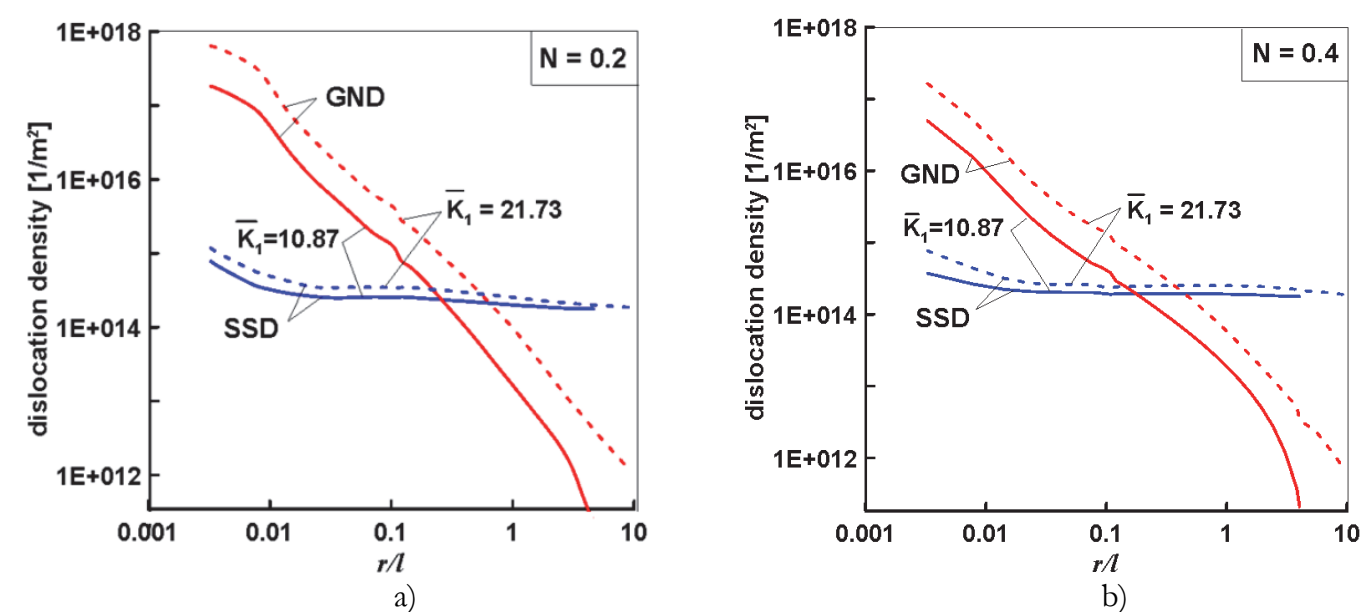

Figure 4: Comparison SSD and GND behavior versus crack tip distance as a function of work hardening exponent N. 
Figs. $4 \mathrm{a}$ and $\mathrm{b}$ show the distributions of the densities $\varrho_{G}$ and $\varrho s$ of geometrically necessary dislocations and statistically stored dislocations as a function of the nondimensional crack tip distance $r / l$ ahead of the crack tip at polar angle $\theta=0^{\circ}$. Here $\varrho_{s}$ and $\varrho_{G}$ are related to the uniaxial stress-plastic strain relation and the effective plastic strain gradient and by $\rho_{S}=\left[\sigma_{r f} f\left(\varepsilon^{P}\right) / M \alpha \mu b\right]^{2}$ and $\rho_{G}=\bar{r}\left(\eta^{P} / b\right)$, according to Eqns.(6) and (7), respectively. It can be seen in Fig. 4 that the density $\varrho_{G}$ of geometrically necessary dislocations is large around the crack tip, but it rapidly decreases away from the crack tip. On the contrary, the density $\varrho s$ of statistically stored dislocations is not as large as $\varrho_{G}$ around the crack tip, but it decreases much slower than $\varrho_{G}$ away from the crack tip. This suggests that both $\varrho_{S}$ and $\varrho_{G}$ are important near the crack tip, which is consistent with the conclusion established from Fig. 3 that the significant increase in stress near the crack tip is due to the geometrically necessary dislocations.

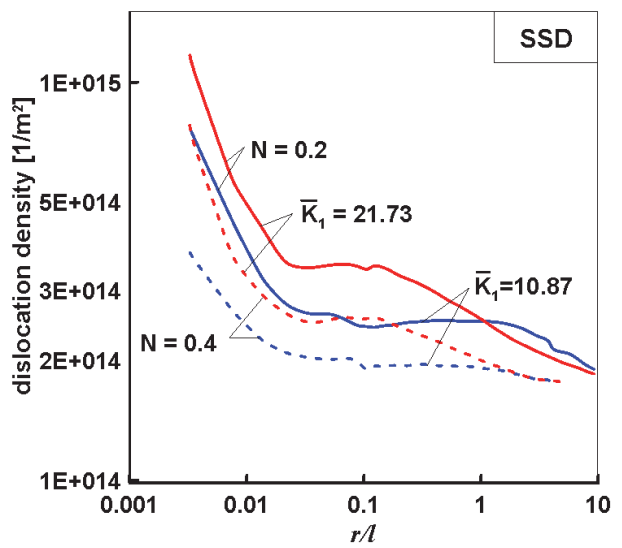

a)

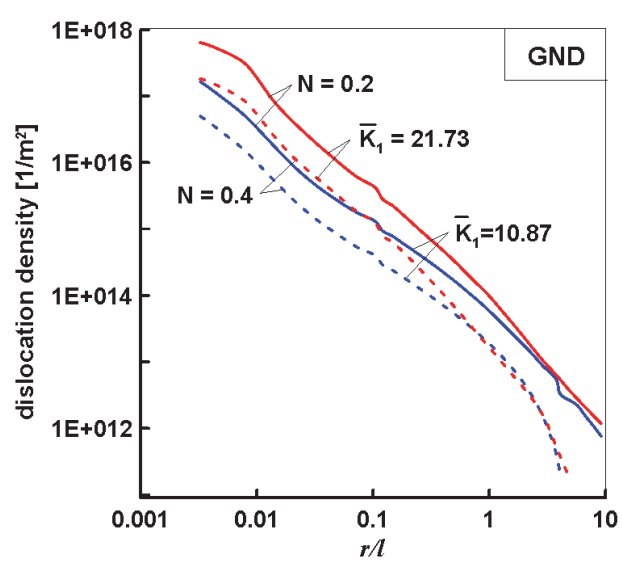

b)

Figure 5: (a) SSD and (b) GND density radial distributions for different loading conditions and plastic material properties.

Figs. 5a and $\mathrm{b}$ provides a separate comparison ahead of the crack tip distributions of the densities $\varrho_{G}$ and $\varrho_{S}$ of geometrically necessary dislocations and statistically stored dislocations for different loading conditions and plastic material properties described by applied stress intensity factors level $\bar{K}_{1}$ and the plastic work hardening exponent $N$ value. As expected, the dislocation density increases with increasing SIF $\bar{K}_{1}$, with higher densities corresponding to a more ductile material at $N=0.2$. Once the distance to the crack tip is less than $0.01 r / l$, the statistically stored dislocations (SSD) density $\varrho$ s predicted by CMSG plasticity rapidly increases (Fig.5a), while the density $\varrho_{G}$ of geometrically necessary dislocations (GND) is monotonously decreases away from the crack tip (Fig.5b).

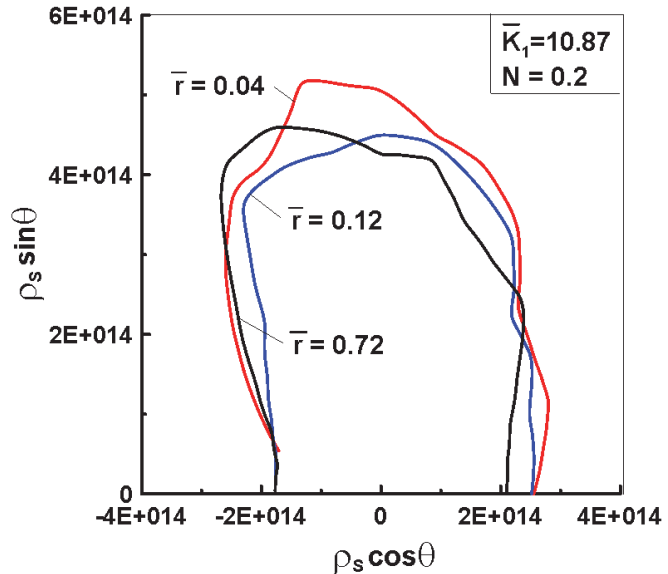

a)

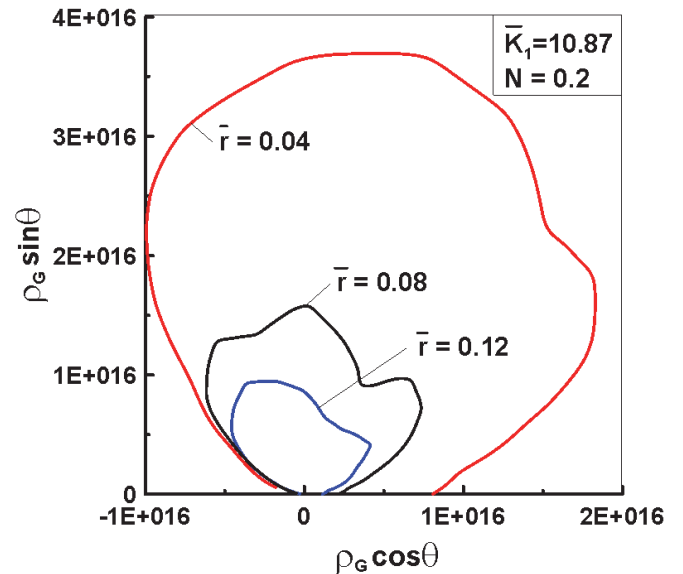

b)

Figure 6: Contour plots of dislocation densities (a) SSD and (b) GND.

Figs. $6 \mathrm{a}$ and $\mathrm{b}$ show the contour plots of the densities $\varrho_{s}$ and $\varrho_{G}$ of statistically stored dislocations and geometrically necessary dislocations as a function of the specified the crack tip distance values that is normalized by the internal material length $l$. Dimension plots of $\rho$ can be shown by considering $x=\rho \cos \theta$ and $y=\rho \sin \theta$. First, Fig. 6 shows the angular 
stress variations at different $r / l$ values. Angular plots are shown for four different dimensionless values of the crack tip distance ranging from 0.04 to 0.72 . The applied stress intensity factor is $\bar{K}_{1}=10.87$, the intrinsic parameter value is $l=5$ $\mu \mathrm{m}$, and the plastic work hardening exponents is $N=0.2$. In Fig.6a, the angular statistically stored dislocations densities $\varrho s$ distributions show a very small sensitivity to the crack tip distance. Fig. 6b shows how the contour plots of the geometrically necessary dislocations densities $\varrho_{G}$ change shape and decrease in size with a gradual increase in the crack tip distance $r / l$.

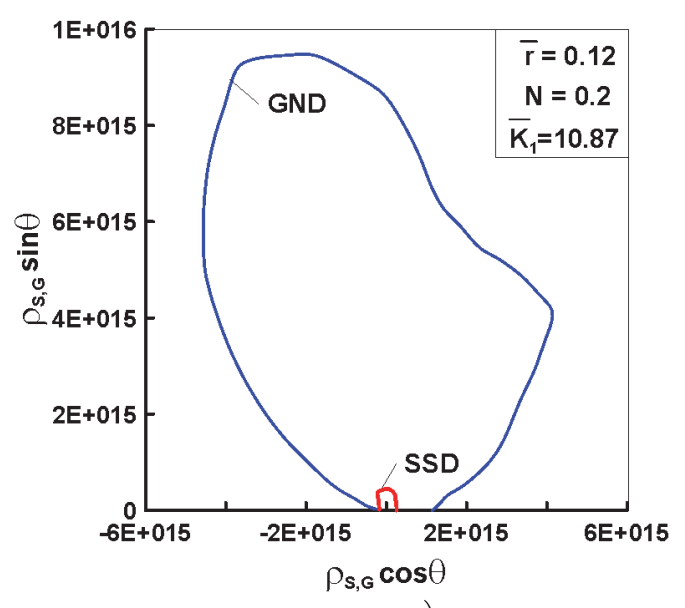

a)

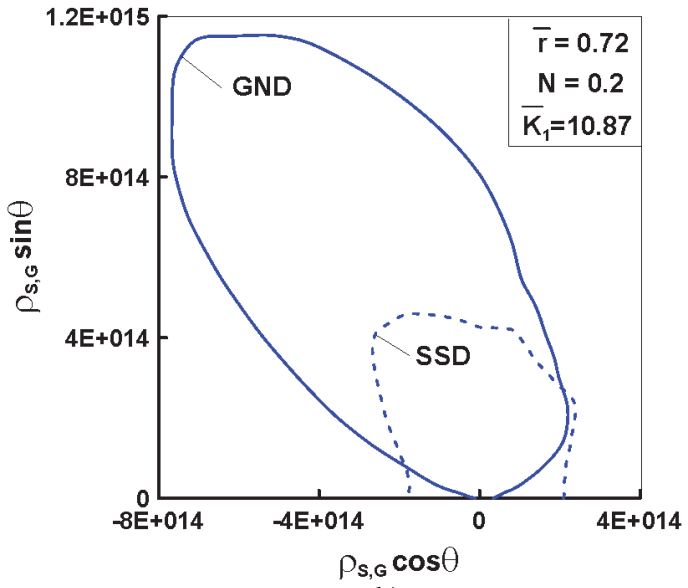

b)

Figure 7: Comparison of contour plots for SSD and GND densities.

Recall that Fig. 3 shows that strain gradient effects become important within a dominated zone at the distance ranging from $0.003(r / l)$ to $0.3(r / D)$ of the crack tip, depending on the strain hardening exponent. Therefore, it is useful to analyze behavior of the dislocation density within this dominated zone. To this end Fig.7 represents a comparison of the contour plots of the SSD and GND densities $\varrho_{S}$ and $\varrho_{G}$ as a function of the dimensionless crack tip distance $r / l$. In Fig.7a the dislocation density distribution within a small distance to the crack tip $r / l \leq 0.12$ indicates that the density $\varrho_{G}$ of GND around a crack tip is significantly larger than the density $\varrho_{s}$ of SSDs along all contours. This is because the strain gradient becomes more singular than the strain near the crack tip and dominates the contribution to the flow stress. However, as it follows from Fig. $7 \mathrm{~b}$, when as $r / l$ gets larger than 0.1 the effect of the plastic strain gradients leads to situation when the densities $\varrho_{s}$ and $\varrho_{G}$ of statistically stored dislocations and geometrically necessary dislocations being the same order of magnitude. Interestingly, the results shown in Fig. 7b, indicates that in the plane ahead of the crack tip $\left(\theta=0^{\circ}\right)$ the density $\varrho_{S}$ of SSD is larger than the density $\varrho_{G}$ GNDs.

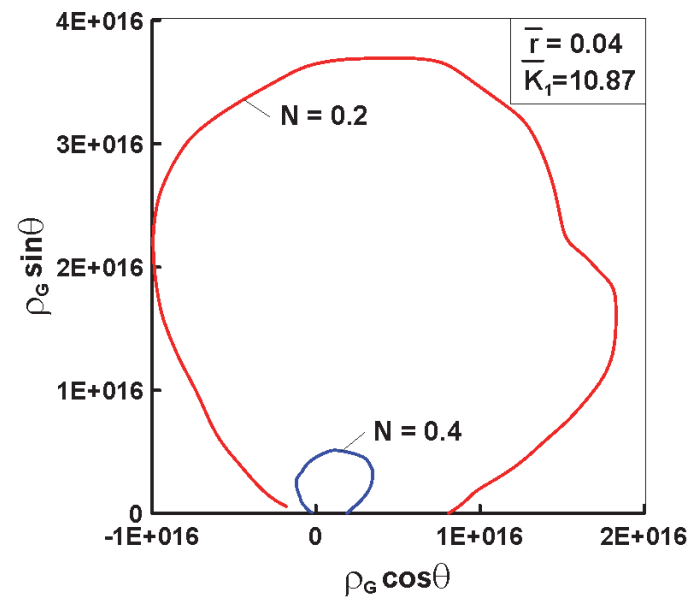

a)

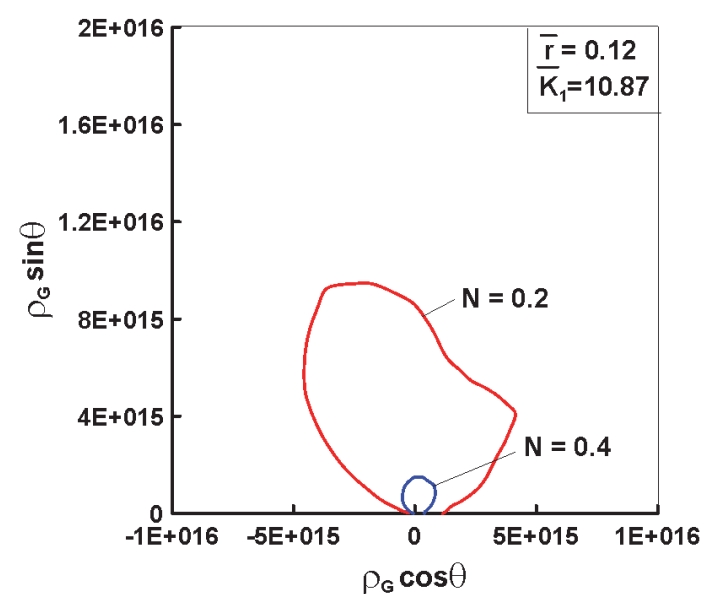

b)

Figure 8: Contour plots of GND densities as a function of plastic work hardening exponent and crack tip distance. 
The sensitivity of the angular geometrically necessary dislocations density distributions to the plastic material properties is investigated in Fig. 8. The calculated angular distributions of the GND density $\varrho_{G}$ are plotted in Fig. 8 for plastic work hardening exponents of $N=0.2$ and 0.4 for the dimensionless crack tip distance $r / l=0.04$ and 0.12 . The applied stress intensity factor is $\bar{K}_{1}=10.87$, the intrinsic parameter value is $l=5 \mu \mathrm{m}$. Figs. $8 \mathrm{a}$ and b show that the difference between the contour plots in strain-gradient plasticity is significant, especially for $N=0.2$, and this difference gradually disappears with an increase in the degree of hardening in the order of transition from plasticity to elasticity. Focusing on the CMSG plasticity results, it is evident that the dislocation density behavior at the crack tip is the result of the combined influence of plastic strain gradients and plastic material properties of the material. The comparison of these the GND density $\varrho_{G}$ variations to each other as a function of the radial coordinate $r / l$ conform very strong sensitivity to the crack tip distance, in agreement with expectations.

During the past two decades it is found that, conventional plasticity lacks an intrinsic length scale and hence cannot predict the size effects observed in experiments. Therefore, it was pointed out the importance of the mesoscale plasticity concepts based on the Taylor model of dislocation hardening and the need to develop a strain gradient plasticity theory with an intrinsic material length scale. This is a significant challenge and should be collectively tackled by wide spread both experimental and numerical investigations. The analysis of the SSD and GND densities $\varrho_{S}$ and $\varrho_{G}$ contributions according to the flow stress constitutive Eqn. (10) presented in this study defines the interrelated participation of a set of governing parameters such as $N, l, r / l$ and $\bar{K}_{1}$ in achieving the general effect of increasing stresses within the framework of the theory of gradient plasticity as compared to the conventional approach.

\section{CONCLUSIONS}

A $\mathrm{s}$ a consequence of the strain-gradient contribution, FE results show a significant increase in the magnitude and the extent of the difference between the crack tip stress fields of CMSGP and conventional HRR theories when the material length parameter is considered. The stress level in this field is three or more times higher than that in the HRR field within a zone on the order of microns around the crack tip. The contour plots of the dislocation densities clearly indicate that both geometrically necessary dislocations and statistically stored dislocations are important around the crack tip. The strain gradient effect associated with geometrically necessary dislocations is responsible for the significant stress increase around the crack tip. The sensitivity of the considered parts of dislocation densities to the coupled effects of the plastic work hardening exponent $N$ and the crack tip distance normalized by material length scale $l$ is established.

\section{ACKNOWLEDGMENT}

he authors gratefully acknowledge the financial support of the Russian Science Foundation under the Project 2019-00158.

\section{REFERENCES}

[1] Fleck, N.A., Hutchinson, J.W. (1993). A phenomenological theory for strain-gradient effects in plasticity, J. Mech. Phys. Solids, 41, pp. 1825-1857. DOI:10.1016/0022-5096(93)90072-N.

[2] Fleck, N.A., Hutchinson, J.W. (1997). Strain gradient plasticity, Adv. Appl. Mech., 33, pp. 295-361. DOI:10.1016/S0065-2156(08)70388-0.

[3] Fleck, N.A., Muller, G.M., Ashby, M.F., Hutchinson, J.W. (1994). Strain gradient plasticity: theory and experiment, Acta Metal. Mater., 42, pp. 457-487. DOI:10.1016/0956-7151(94)90502-9.

[4] Huang, Y., Zhang, L., Guo, T.F., Hwang, K.C. (1995). Near-tip fields for cracks in materials with strain gradient effects, Proc. IUTAM Symposium on Nonlinear Analysis of Fracture (Edited by J.R.Wills), Kluwer Academic Publishers, Cambridge, England, pp. 231-242.

[5] Huang, Y., Zhang, L., Guo, T.F., Hwang, K.C. (1997). Mixed mode near-tip fields for cracks in materials with strain gradient effects, J. Mech. Phys. Solids., 45, pp. 439-465. DOI:10.1016/S0022-5096(96)00089-0. 
[6] Xia, Z.C, Hutchinson, J.W. (1996). Crack tip fields in strain gradient plasticity, J. Mech. Phys. Solids., 44, pp. 16211648. DOI:10.1016/0022-5096(96)00035-X.

[7] Nix, W.D., Gao, H. (1998). Indentation size effects in crystalline materials: a law for strain gradient plasticity, J. Mech. Phys. Solids., 46, pp. 411-425. DOI:10.1016/S0022-5096(97)00086-0.

[8] Gao, H., Huang, Y., Nix, W.D., Hutchinson, J.W. (1999). Mechanism-based strain gradient plasticity-I, Theory. J. Mech. Phys. Solids., 47, pp. 1239-1263.

[9] Huang, Y., Qu, S., Hwang, K.C., Li, M., Gao, H. (2004). A conventional theory of mechanism-based strain gradient plasticity, Int. J. Plast., 20, pp. 753-782. DOI:10.1016/j.ijplas.2003.08.002

[10] Jiang, H., Huang, Y., Zhuang, Z., Hwang, K.C., 2001. Fracture in mechanism-based strain gradient plasticity. J. Mech. Phys. Solids 49, 979 - 993. DOI: 10.1016/S0022-5096(00)00070-3

[11] Shi, M., Huang, Y., Jiang, H., Hwang, K.C., Li, M. (2001). The boundary-layer effect on the crack tip field in mechanism-based strain gradient plasticity, Int. J. Fract., 112, pp. 23-41. DOI: 10.1023/A:1013548131004.

[12] Qu S, Huang Y, Jiang H, Liu C. (2004). Fracture analysis in the conventional theory of mechanism-based strain gradient (CMSG) plasticity, Int. J. Fract., pp. 199-220. DOI: 10.1023/B:FRAC.0000047786.40200.f8

[13] Martínez-Pañeda, E., Betegon, C. (2015). Modeling damage and fracture within strain-gradient plasticity, Int. J. Solids Struct., 59, pp. 208 -215. DOI:10.1016/j.ijsolstr.2015.02.010

[14] Martínez-Pañeda, E., Niordson, C.F. (2016). On fracture in finite strain gradient plasticity, Int. J. Plast., 80, pp. 154167. DOI: $10.1016 /$ j.ijplas.2015.09.009

[15] Martínez-Pañeda, E., Natarajan, S., Bordas, S. (2017). Gradient plasticity crack tip characterization by means of the extended finite element method, Comp. Mech., 59, pp. 831-842. DOI: 10.1007/s00466-017-1375-6

[16] Martínez-Pañeda, E., Fleck, N.A. (2019). Mode I crack tip fields: Strain gradient plasticity theory versus J2 flow theory, Europ. J. Mech.A Solids., 75, pp. 381 -388. DOI: 10.1016/j.euromechsol.2019.02.009

[17] Gao, H., Huang, Y. (2003). Geometrically necessary dislocation and size-dependent plasticity, Scripta Materialia., 48, pp. 113-118. DOI: 10.1016/S1359-6462(02)00329-9

[18] ANSYS Mechanical APDL Theory Reference Release 14.5, ANSYS, Inc. Southpointe, 275 Technology Drive, CanonBurg, PA 2012. 